\title{
HyCo - An Authoring Tool to Create Semantic Learning Objects for Web-Based E-learning Systems
}

\author{
Francisco J. García ${ }^{1,2}$, Adriana J. Berlanga², Maria N. Moreno ${ }^{1}$, Javier García ${ }^{2}$, and \\ Jorge Carabias ${ }^{1}$ \\ ${ }^{1}$ Department of Computer Science - University of Salamanca (Spain) \\ JORGECARABIAS@ telefonica.net \\ ${ }^{2}$ Institute of Educational Sciences - University of Salamanca (Spain) \\ \{fgarcia, solis13, mmg, carrasco\}@usal.es
}

\begin{abstract}
In this article we introduce HyCo (Hypertext Composer), an authoring tool devoted to create semantic learning objects. This authoring tool uses learning technology standards or specifications to save these semantic objects, which will be delivered in Web e-learning environments as encapsulated packages in order to ensure their reusability, interoperability, durability and accessibility. These learning objects are closed to the Semantic Web field because they combine hypermedia and semantic capabilities. Our research work is directed to use these semantic learning objects in order to define learning domains for an Adaptive Learning Environment. The aim of this system is to provide an e-learning environment where teachers have tools to create didactic materials and students carry out their knowledge acquisition through the most suitable adaptive learning technique giving the student's characteristics, the learning activities provided, and the learning objects' features.
\end{abstract}

Keywords: Hypermedia Authoring Tools; Semantic Web Applications; Learning Technologies Standards; IMS; E-Learning.

\section{Introduction}

The use of Internet as an instructional media not only has brought new ideas and thoughts around learning and teaching, but also a new conception about learning elements through WWW: they should be reusable, interoperable, durable and accessible.

To accomplish these requirements, several education institutions had incorporated information and communication technologies in the learning and teaching process in order to increase the quality, efficiency, and dissemination of the education. Consequently, learning domains have been passed thru a reconfiguration process where defining metadata for learning objects has a central role. Metadata guarantees interoperation, reusability, and interchange among e-learning systems, as well as a cost-effective development.

\footnotetext{
* This study was partly financed by the Regional Government of Castile and Lion through research project SA017/02. Also, it is supported by the European Union Project ODISEAME, ref. EUMEDIS B7-4100/2000/2165-79 P546.
} 
Several LTS (Learning Technology Standards) have been defined, which are agreements about the characteristics a learning element should have in order to be compatible, interchangeable and interoperable into other learning systems [9].

If web-based e-learning systems fulfill a LTS, they are able to work with other systems (interoperability), follow-up information about learners and contents (manageability), generate learning objects that are usable in other contexts (reusability), and avoid obsolescence (durability). From students' point of view, standards ensure they get the right content at the right time (accessibility) and obtain a variety of knowledge resources (interchange of learning objects).

In our research work, we are interesting in defining SLOs (Semantic Learning Objects) that will be compliant with these LTSs and will be integrated or deployed in an ALE (Adaptive Learning Environment). The aim of an ALE system is to provide an e-learning environment where teachers have tools to create didactic materials and students carry out their knowledge acquisition through the most suitable adaptive learning technique giving the student's characteristics, the learning activities provided, and the learning objects' features [1].

The proposed ALE architecture is composed of five subsystems: Learning Domain Model, Adaptive Model, Student Model, Adaptive Meta-model, and Deliver and Packing Model, as it is shown in Figure 1.

The system pretends to be an open tool that

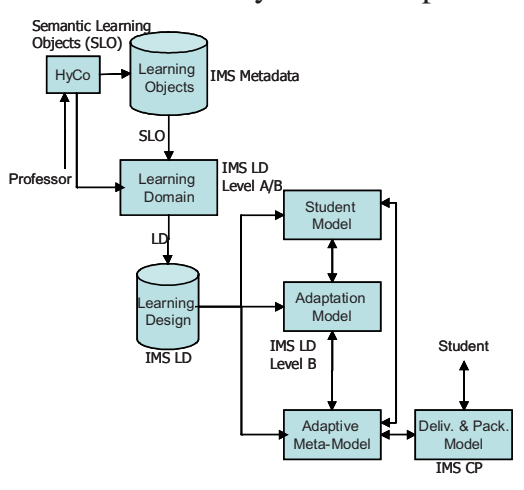

Fig. 1. ALE Architecture differentiates between educative contents and learning process. The ALE system structures its semantic elements following the IMS specifications. Namely, to describe Learning Objects it uses IMS Metadata [6], to describe the Deliver and Packing Model it uses IMS Content Packaging (IMS CP) [4], and to describe the learning domain model, it uses IMS Learning Design (IMS LD) [5].

The rest of the paper is organised as follows. Section 2 gives a general introduction of the HyCo authoring tool. Section 3 is focused on the definition of the SLOs in HyCo. Finally, section 4 provides remarks and further work.

\section{An Overview of the HyCo Authoring Tool}

HyCo [3] is a powerful authoring tool for educational purposes; this means that an author can create hypermedia educational resources with it. But also the same tool could be used to access to the created contents in a read-only mode by a student or reader.

HyCo is a multiplatform tool. It does not force to use one concrete platform. The idea is that if we want the teachers use it, they should work in the context they feel good. The actual version of HyCo works in the wider range of operating systems, for this reason Java 2 Standard Edition technology was chosen as development base.

The main goal of the HyCo is the creation of educational contents, but trying to 
achieve an independence of the final format publication. There exits a clear separation between the contents and its presentation. This way the educator writes the contents once, and reuses them every time he/she needs. In order to achieve this goal, $\mathrm{HyCo}$ tool uses an internal XML-based format [2] to store the educational contents of the produced electronic books. Precisely, the HyCo XML-based format allows the introduction of the LTSs in this authoring tool, specifically HyCo supports IMS specifications [4, 5, 6] and EML (Educational Modelling Language) [8].

The fact of separating the content and the presentation forces to offer to the authors a way to generate an independent result of the authoring tool. In this way HyCo has an output gallery that supports HTML, PDF, TXT, RTF, SVG and PS output formats.

Following pedagogical criteria, HyCo reproduces the process that an author would follow to create a linear educational resource, but it channels it, at the same time as it organizes it, through the metaphor of the content index, adding the facilities for including multimedia elements as well as hyperlinks. This way a hierarchical structure is obtained that guides us in our creative process, which would consist in associating contents with each index entry, an index that may vary as the contents take shape, by inserting, eliminating or changing entries. In a nutshell, then, HyCo faithfully reproduces the process previously explained. This indexed or tree structure facilitates the authoring of the hypertext, but having only an index as navigation tool is not acceptable in order to create real pedagogical hypermedia resources where the student may construct its own knowledge. This way, the hyperdocuments should be designed in such a way as to encourage the readers to see the same text in as many useful contexts as possible. This means placing texts within the contexts of other texts, including different views of the same text [7]. For this reason HyCo also allows associating links to the multimedia elements that compose an index entry, i.e. a hypertext node. Thus, the hypertext can be followed by its index-structure, but when a node is selected, the reader may choose navigating by an existing link. Thus, HyCo documents combine both content index and Web-like structures.

\section{Definition of Learning Resources in $\mathrm{HyCo}$}

When organizations, schools and teachers started to use the Web as an instructional media, almost the only way to publish educational contents was in HTML format, where e-learning elements were presented without any division between content and its meaning. This syntactic presentation prevents to automatically extract data, not to mention the definition of learning elements were as heterogeneous as existing elearning designs. In this context, interoperability, reusability, and interchange among e-learning systems are impossible. Moreover, the idea of a cost-effective e-learning development is far away.

Several organizations have been working to define specifications and standards to design instructional elements, known as Learning Technologies Specifications. EML and IMS Specifications are LTSs that are now supported in HyCo authoring tool.

As we stated before, HyCo is an authoring tool to create SLOs, which are the learning resources that will be available in the instructional design process that defines the ALE learning domain. 


\subsection{Definition of Learning Resources}

To create the learning domain model, the first step is to generate SLOs. Every SLO should be compliant with IMS Metadata [6]. Every section of every educational resource or e-book created in HyCo can be converted to a SLO.

To do it, HyCo executes a two-step process where the first step is an automatic process, while the second step is a manual process. In the automatic process, HyCo sets all the IMS Metadata elements that can be inferred from other data or that are liable to have default values.

Once this process is over, HyCo executes the manual process where it presents to the user the elements that can not be automatically generated and/or require reexamination, modification, or addition.

When the two-step process is finished a XML file is generated for each new SLO (each one of them corresponds to each educational resource, section, or subsection) and stored in an IMS Metadata SLO repository. This repository will allow us to have learning objects that can be attached to learning activities of the learning designs created in ALE.

\subsection{Definition of Learning Components}

The learning components design includes the definition of roles, learning activities and learning activities sequences.

Two steps are needed to define the learning components. First the addition and definition of roles and learning activities, and then connect these elements by means of an activity sequences.

The definition of roles includes elements as title, metadata and information. The definition of learning activities includes elements as title, metadata, learning objectives, prerequisites, description, feed back description, and so on. Also SLO can be included.

In order to ensure efficiency and simplicity in the authoring process, repositories of learning objects and learning designs, as well a set of selectors and creators will be provided. For example, to define learning activities the author can choose one learning object (i.e. SLO) from its repository and use selectors for describe elements as metadata, prerequisites, and learning objectives. Moreover, these selectors will be used reiteratively within the different definitions, where the same elements exist. The most representative case of this is the metadata selector that will be used when defining roles, learning activities and sequences of activities.

In addition, in the creation of learning activities, ALE will be able to propose learning objects taking into account the metadata stored in other elements as prerequisites, objectives, and so on.

After the author has defined the learning components, the next phase is to design the learning method where conditions and attributes will be delimited. Finally, the learning components and the learning method have to be integrated into the definition of the learning design, where general prerequisites and objectives are added, as well as roles, activities (that group learning activities and sequences), and the learning method. 


\section{Conclusions and Further Work}

In this paper we have introduced HyCo as an authoring tool that allows the definition of both learning resources and learning components or SLOs, i.e. semantic educational resources based on XML specifications, which could be delivered in diverse Learning Management Systems. Specifically, HyCo supports EML and IMS.

The success of the HyCo authoring process has been proved with three educational web-based systems. Two of them are drafts devoted to test the authoring tool, one about computer history and other one about a software engineering course. But the third one is a complete electronic book in hypermedia format about cardiovascular surgery that is formed by 14 chapters, more than 500 sections and over 1000 images. This book is successfully used in the lectures of this subject in the University of Salamanca.

$\mathrm{HyCo}$ is an open system in which too much further work is going to be done, especially in adaptive hypermedia systems. Now the next step is to make the necessary changes and modifications to $\mathrm{HyCo}$ in order to turn it into the learning domain authoring tool of the ALE System.

Also, we are working in defining two more phases for the learning domain. Namely, a definition of a learning style theory and the definition of the adaptive rules the ALE system will follow. The first will be necessary to setup the students' characteristics that will be considered. The second will be helpful to define the adaptation rules the system will take into account to perform the adaptation of contents and links.

\section{References}

[1] Berlanga, A., Morales, E., García, F. J.: Learning Technology Standards: Semantic Objects for Adaptive Learning Environments. In A. Méndez, J. A. Mesa, J. Mesa (Eds.), Advances in Technology-Based Education: Toward a Knowledge-Based Society. Proceedings of the $2^{\text {nd }}$ International Conference on Multimedia and Information \& Communication Technologies in Education m-ICTE2003. Consejería de Educación, Ciencia y Tecnología de la Junta de Extremadura. (2003) Vol. II, 860-864.

[2] Bray, T., Paoli, J., Sperberg-MacQueen, C. M., Maler, E., Yergeau, F. (Eds.): Extensible Markup Language (XML) 1.0 (Third Edition). World Wide Web Consortium. http://www.w3.org/TR/2004/REC-xml-20040204. (2004).

[3] García, F. J., García, J.: Educational Hypermedia Resources Facilitator. Computers \& Education. In press. (2004).

[4] IMS: IMS Content Packaging. http://www.imsglobal.org/content/packaging/index.cfm. (2003).

[5] IMS: IMS Learning Design Specification. http://www.imsglobal.org/learningdesign/index.cfm. (2003).

[6] IMS: IMS Learning Resource Metadata Specification. http://www.imsglobal.org/metadata/index.cfm. (2003).

[7] Jones, R. A., Spiro, R.: Imagined Conversations: The Relevance of Hypertext, Pragmatism, and Cognitive Flexibility Theory to the Interpretation of "Classic Texts" in Intellectual History. In D. Lucarella, J. Nanard, M. Nanard, P. Paolini (Eds.), Proceedings of the $4^{\text {th }}$ ACM Conference on Hypertext - ECHT'92. ACM Press (1992) 141-148.

[8] Koper, R.: Modelling Units of Study from a Pedagogical Perspective. The Pedagogical Metamodel behind EML. http://eml.ou.nl/introduction/docs/ped-metamodel.pdf. (2001).

[9] Wiley, D.: Connecting Learning Objects to Instructional Design Theory: A Definition, a Metaphor, and a Taxonomy. In D. Wiley (Ed.), The Instructional Use of Learning Objects. On line version: http://reusability.org/read/chapters/wiley.doc. (2000). 